\title{
Importancia de la heterogeneidad ambiental en la ecología de plantas carnívoras mediterráneas: implicaciones para la conservación
}

\author{
Environmental heterogeneity and the ecology of carnivorous plants: implications for \\ conservation
}

REGINO ZAMORA

Grupo de Investigación Ecología Terrestre, Departamento de Biología Animal y Ecología, Facultad de Ciencias, Universidad de Granada, E-18071 Granada, España; e-mail: rzamora@goliat.ugr.es

\section{RESUMEN}

En la cuenca mediterránea, la mayoría de las plantas carnívoras pertenecientes al género Pinguicula habitan las paredes húmedas de montañas calizas. Estos escenarios rocosos condicionan la ecología de las poblaciones situadas en distintos exposiciones (solana versus umbría). Las diferencias temporales en la floración entre plantas que crecen en distintos microhábitats, unido a las diferencias espaciales en la distribución y abundancia de las especies de polinizadores, provocan barreras al flujo genico vía polen entre microhábitats soleados y umbríos. Estos mecanismos ecológicos pueden actuar sinérgicamente con otros factores que limitan el flujo génico, como distancia geográfica y la compleja orografía de las montañas, favoreciendo la diferenciación local. Las poblaciones de Pinguicula vallisneriifolia muestran también diferentes abundancias y estructuras demográficas dependiendo del microhábitat donde crecen las plantas. El carácter perenne y la reproducción asexual de esta planta le permite ralentizar la extinción poblacional, incluso en ausencia de reclutamiento via plántula en los lugares secos y soleados. Sin embargo, con la actual tendencia hacia una mayor aridez, los escasos micrositios adecuados para la germinación y establecimiento de plántulas se desplazan hacia los sectores de las paredes más umbríos y húmedos, pero donde el desarrollo reproductivo está limitado por la escasez de luz. Para la conservación de esta planta carnívora hay que llevar a cabo medidas de manejo para mantener un número mínimo de nichos de regeneración efectivos. La conservación de $P$. vallisneriifolia pasa por mantener la riqueza de escenarios ecológicos (i.e., mantener poblaciones de sol versus de sombra), como parte de la variabilidad total a conservar, no solo geográfica, sino también de escenario microclimático.

Palabras clave: heterogeneidad ambiental, ecología y conservación, planta carnívora, interacciones planta-animal, estructura demográfica.

\begin{abstract}
Most carnivorous plants belonging to the Pinguicula genus inhabit mountains in the Mediterranean basin. Pinguicula vallisneriifolia inhabits wet limestone rock walls and cliffs, being able to reproduce by seeds and asexually (by stolons and axillary buds). The complex orography of Mediterranean mountains favors sunny (hot and dry) and shady (cool and wet) contrasting expositions. Along the sun-shade gradient, there were variations in plant flowering phenology, as well as in animal species that interacted with the plants (prey, pollinators and kleptoparasites), even at a very restricted spatial scale. The ecological consequence is that the marked spatial variability in abiotic conditions results in a site-specific mosaic of plant-animal interactions, and hence of selective pressures of animals upon plants. Plant populations have also different demographic structures depending on the microhabitat (sunny, dry versus wet, shady). As a result, different demographic patterns appear on different localities or different habitats within the same locality: populations living in moist, shady patches show high proportions of seedlings as plants reproduce both sexually and asexually, resulting in viable populations. On the contrary, plants living in dry, sunny rocky substrates, despite sexual and asexual reproduction, have no seedling recruitment because of wet microsite limitation. These populations are, therefore, dominated by vegetative individuals. Practical guiadance are provided in order to preserve these microclimatically structured populations.
\end{abstract}

Key words: abiotic heterogeneity, ecology and conservation, canivorous plant, plant-animal interactions, demographic structure. 


\section{INTRODUCCIÓN}

La mayoría de las plantas carnívoras aparecen restringidas a hábitats pobres en nutrientes, que permanecen muy húmedos y soleados durante el período de crecimiento, como turberas y zonas encharcadas (Givnish 1989). A pesar de la rareza de este tipo de hábitats húmedos en las regiones con clima mediterráneo y semiárido, se conocen muchas especies carnívoras del género Pinguicula tanto en la cuenca mediterránea (Casper 1972) como en México y California (Givnish 1989, Studnika 1991).

En la cuenca mediterránea, las plantas carnívoras pertenecientes al género Pinguicula habitan preferentemente en paredes y taludes de montaña. La compleja orografía de las montañas mediterráneas favorece la existencia de exposiciones contrastadas de solana y umbría, cálida y seca la primera, fresca y húmeda la segunda. Estos escenarios contrastados pueden condicionar la ecología de las poblaciones situadas en distintas exposiciones, ya que, incluso a una escala espacial reducida, la heterogeneidad ambiental provoca diferenciación morfológica y fisiológica en plantas, condicionando tanto su fenología, estructura demográfica de las poblaciones y evolución (Linhart \& Grant 1996). La heterogeneidad abiótica puede también condicionar el resultado de las interacciones planta-animal (Bronstein 1994), y muy particularmente, la distribución y abundancia de los insectos polinizadores, a una escala de resolución espacial muy fina (Herrera 1995b, 1997, Zamora 1995, 1999). Ambos aspectos son relevantes tanto desde una aproximación puramente teórica y multidisciplinar, como desde un punto de vista aplicado. En el presente ensayo, expongo un análisis que pretende poner de manifiesto algunos aspectos de especial trascendencia para diseñar planes de conservación y manejo de Pinguicula vallisneriifolia, usando esta planta carnívora como modelo de organismo que vive en hábitats ecológicamente muy heterogéneos.

Pinguicula vallisnerifolia es una planta insectívora endémica que crece en paredes rezumantes de montañas calizas del sureste de la Península Ibérica (Zamora et al. 1996). Lo inaccesible de su hábitat natural le permite librarse de la mayoría de los factores que inciden negativamente en otras especies endémicas, como herbivorismo, fuego y recolección (Blanca et al. 2000).

Pinguicula vallisnerifolia presenta poblaciones aisladas a lo largo de su área de distribución geográfica, con tamaño variable (de menos de 100 a más de 1.000 plantas reproductoras). Sin embargo, dado el grado de fragmentación de sus poblaciones, el tamaño reducido de muchas de ellas, y su dependencia por el agua, la aridez actual de la Cuenca Mediterránea hace que esta especie sea vulnerable a la extinción, como le ocurre a muchas otras plantas endémicas asociadas a los hábitats húmedos en el Mediterraneo. Es urgente por tanto identificar los factores que determinan la viabilidad de los fragmentos poblaciones existentes bajo condiciones ecológicas muy heterogéneas, con el fín de diseñar planes de conservación realistas a medio y largo plazo (Falk \& Holsinger 1991, Schemske et al. 1994).

\section{HISTORIA NATURAL}

Pinguicula vallisnerifolia es una herbácea perenne que crece y se reproduce a lo largo de un amplio gradiente de radiación luminosa, tolerando el sombreado permanente producido por las paredes extraplomadas, pero creciendo también a pleno sol, siempre y cuando tengan una adecuada disponibilidad de agua durante todo el período de crecimiento vegetativo (abril-septiembre). Las plantas pueden colonizar espacios vacíos en suelos saturados de agua, inmediatamente adyacentes a la pared de roca donde se encuentra el resto de la población, o en los márgenes de una fuente o arroyo, siempre que no exista otra vegetación competidora. De hecho, P. vallisneriifolia es la primera planta colonizadora que aparece en los espacios abiertos húmedos tras una perturbación. Las plantas pueden aparecer incluso en paredes secas, aunque siempre enraizando en pequeñas grietas y/o acúmulos de materia orgánica que conservan humedad.

Al contrario que las restantes especies del género, $P$. vallisnerifolia desarrolla dos tipos de hojas glandulares durante su crecimiento vegetativo. Las primeras 5-7 hojas se desarrollan durante la primavera, y forman una roseta basal. Posteriormente, cada planta puede desarrollar entre 4 y 10 hojas distales, mucho más largas que anchas, y hasta de $30 \mathrm{~cm}$ de longitud, durante el período mayo-agosto (Zamora et al. 1996). Las hojas distales se vuelven senescentes en septiembre, con el inicio del desarrollo de la yema central invernal y las yemas axilares. $P$. vallisnerifolia se reproduce asexualmente, por medio de estolones (verano) y yemas axilares (otoño).

La floración se produce simultáneamente con el desarrollo de las primeras hojas distales (abrilmayo). Un individuo reproductor puede producir hasta 10 flores, aunque lo normal es entre 1 y 4 . Pinguicula vallisnerifolia presenta flores zigomorfas, con espolón, de hasta más de $2 \mathrm{~cm}$ de altura, variando la coloración general de la corola 
desde tonos violetas fuertes a coloraciones rosadas, a veces casi completamente blancas (Zamora et al. 1996).

La fenología de floración depende de la altitud a la que se encuentre la población (rango altitudinal: 600-1.800 m), y, sobre todo, de la orientación de la pared rocosa (solana, umbría) donde crecen las plantas. El resultado es que poblaciones situadas a la misma altitud, pero en exposiciones opuestas (solana vs umbría) apenas solapan sus fenologías (Ellison et al. en prensa).

\section{Biología de la reproducción}

Las flores de $P$. vallisneriifolia son completamente autocompatibles. Sin embargo, en condiciones naturales no se da autogamia espontánea, por lo que la planta necesita a los polinizadores como vectores polínicos. Las flores de $P$. vallisneriifolia sometidas a un regimen natural de polinizadores tienen un éxito de fructificación muy variable (23-100\%). Las poblaciones que crecen en paredes soleadas presentan un mayor éxito de fructificación, mientras que, en las poblaciones situadas en ambientes umbríos la proporción de cápsulas es menor (Zamora 1999). Sin embargo, la adición experimental de polen asegura éxitos de fructificación del $100 \%$ tanto en los hábitats soleados como umbríos, lo que indica claramente que el éxito reproductivo de $P$. vallisneriifolia está muy limitado por la escasez de vectores polínicos (polinizadores), especialmente en los sectores umbríos (Zamora 1999).

La fructificación ocurre de junio a agosto, y las semillas son producidas dentro de un fruto capsular, que se inclina hacia la pared, abriendo hacia ella (geoautocoria). Sin embargo, debido a su pequeño tamaño, (aproximadamente $12 \mu \mathrm{g}, 1$ $\mathrm{mm}$ de longitud), y al hecho de que las plantas pueden crecer en lo alto de las paredes rocosas, la semillas pueden ser dispersadas por el viento.

\section{ESTRUCTURACIÓN MICROCLIMÁTICA DE LAS INTERACCIONES PLANTA-ANIMAL}

Como cualquier otra angiosperma, $P$. vallisneriifolia puede ser comida por herbívoros, y polinizada por insectos (Givnish 1989, Juniper et al. 1989). A estas interacciones (herbivorismo, polinización), comunes con cualquier otra planta, una planta carnívora añade un plus adicional de diversidad, ya que se comporta como un depredador (sit-and-wait predator), atrapando a los insectos de pequeño tamaño que se posan en sus hojas (usualmente de menos de $5 \mathrm{~mm}$ de longitud en el caso de P. vallisneriifolia, Zamora 1995). Las secreciones glandulares permiten la digestión de la presa, y los nutrientes extraidos de la misma son fundamentales para el crecimiento y reproducción de la planta (Zamora et al. 1997, 1998). Esta interacción trófica puede ademas volverse mucho más barroca, ya que aparecen animales oportunistas cleptoparásitos (babosas y lagartijas fundamentalmente) que sacan partido de los restos animales atrapados en la hojas.

Todos los animales con los que interactúa la planta (presas, polinizadores y cleptoparásitos) se distribuyen diferencialmente a lo largo del gradiente abiótico (Zamora 1995, 1999, Zamora \& Gómez 1996), lo que añade un nivel adicional de complejidad espacial al ya de por sí diverso sistema de interacciones entre esta planta insectívora y los animales mutualistas y antagónicos con los que interacciona.

Las presas de $P$. vallisnerifolia son insectos alados (fundamentalmente dípteros nematóceros), que se distribuyen diferencialmente en el mosaico espacial de microhábitats soleados y secos versus húmedos y umbríos. Durante el verano (junio-julio) es cuando las plantas tienen todas sus hojas distales completamente desarrolladas y funcionales, y cuando captura más presas. Los dípteros se concentran en los lugares más umbríos, evitando los lugares soleados y secos conforme avanza el caluroso verano mediterráneo. Por el contrario, otros grupos, como tisanópteros y áfidos evitan los lugares umbríos, y son mucho más abundantes en los lugares soleados (Zamora 1995).

Una vez las presas han sido atrapadas, pueden ser robadas de las hojas por una serie de animales cleptoparásitos, cuya distribución espacial también es heterogénea, estando asociada al grado de humedad del sustrato, y al nivel de insolación. Una babosa, Deroceras grimaldii (Agriolimacidae), es el principal animal kleptoparásito en los sectores mas húmedos y umbríos (Zamora \& Gómez 1996), mientras que una lagartija endémica, Algyroides marchi (Lacertidae) es el principal cleptoparásito de las plantas que crecen en los hábitats rocosos soleados (Zamora 1995).

\section{Polinizadores}

Las paredes rocosas donde crecen las plantas, de hasta $100 \mathrm{~m}$ de altura y verticales, o incluso extraplomadas, limitan considerablemente el acceso de los rayos del sol a las plantas, sobre todo si la pared está expuesta al norte. En estas condiciones, las temperaturas permanecen bajas durante el período de floración de $P$. vallisneriffollia 
(abril-mayo), lo que limita la presencia de muchos insectos que no pueden permanecer activos por debajo de determinados umbrales de temperatura (Corbet 1990, Herrera 1995a). Por ello, las flores de $P$. vallisnerifolia reciben muy pocas visitas florales (sobre todo cuando las plantas crecen en lugares umbríos), básicamente de Bombylius sp., Lassioglosum sp., Bombus sp., y pequeños insectos florícolas, como tisanopteros Taeniothrips meridionalis, Thysanoptera) y escarabajos (Eusphalerum scribae, Staphylinidae).

La heterogeneidad en las condiciones abióticas condiciona tanto la fenología de floración de $P$. vallisneriifolia, como la distribución espacial de sus insectos polinizadores. En los microhábitats soleados de poblaciones separadas geográficamente, las plantas comparten una fenología de floración temprana, y las mismas especies de polinizadores. En las microhábitats más umbríos, las plantas tambien comparten una fenología más retrasada (un mes de diferencia, para poblaciones situadas a la misma altitud, pero en exposiciones opuestas). Por otra parte, las flores apenas reciben visitas florales. El resultado es que, aunque aparecen las mismas especies de polinizadores a lo largo de toda el área de distribución geográfica de la planta, la distribución espacial de los insectos es muy heterogénea, apareciendo unas especies (e.g., Bombylius, Lassioglosum, T. meridionalis) en los microhábitats soleados, y otras en los microhábitats umbríos (E. scribae).

Consecuencias evolutivas de la estructuración microclimatica de la interacción plantapolinizador

La variabilidad espacial en las condiciones abióticas puede generar un mosaico espacial de presiones selectivas en la interacción plantapolinizador debido a las diferencias microclimáticas entre poblaciones soleadas y umbrías, y, dentro de población, incluso a escala de microhábitats (parches de la pared soleados versus umbríos, Zamora 1999, Ellison et al. en prensa).

Las diferencias temporales en la fenología de floración entre plantas que crecen en distintos microhábitats, unido a las diferencias espaciales en la distribución y abundancia de las especies de polinizadores, pueden provocar barreras al flujo génico vía polen entre poblaciones con diferente exposición (solana versus umbría), e incluso entre microhábitats soleados y umbríos dentro de la misma población. La diferenciación resultante estaría por tanto provocada por éstos factores ecológicos que pueden actuar sinérgicamente con otros, como la distancia geográfica y la compleja orografía de la montaña, limitando el flujo génico y provocando incluso especiación (Ellison et al. en prensa, véase Schluter 1998 y Johannesson 2001 para ejemplos de especiación ecológica).

Este nivel de variabilidad microclimática existente entre y dentro de poblaciones (con sus escenarios ecológicos de sol y de sombra), afecta también a muchas otras plantas que aparecen en los mismos hábitats rocosos que $P$. vallisneriifolia, pudiendo ser, como ocurre en esta planta carnívora, un factor condicionante de la diversidad de las especies rupícolas. A continuación voy a explorar esta posibilidad, tomando como ejemplo las especies rupícolas de la Península Ibérica, que es uno de de los reservorios principales de biodiversidad en la cuenca mediterránea (Castroviejo 1997). En la Península Ibérica hay alrrededor de 1.200 especies y subspespecies endémicas (Gómez-Campo \& Herranz-Sanz 1993), las cuales representan aproximadamente el $15 \%$ de todos los taxa de la Península Ibérica (alrrededor de 8.000 especies, Castroviejo 1997).

En las montañas mediterráneas, el porcentaje de especies endémicas es muy alto, llegando a niveles superiores al $50 \%$ en algunas montañas del sur peninsular (Blondel \& Aronson 1999). Los hábitats rocosos albergan el porcentaje mayor de especies endémicas de toda la Peninsula (aproximadamente el $57 \%$ del total de plantas con flor, Fernández-Casas \& Ceballos 1982). En el caso de Sierra Nevada, la proporción de especies endémicas en substratos rocosos puede llegar hasta el $80 \%$ (Blanca et al. 1998). Por tanto, en una región muy diversa en especies de plantas, como es el caso de la Península Ibérica, los substratos rocosos son, con diferencia, los puntos más calientes de diversidad. Es evidente que éste nivel inusual de diversidad debe atribuirse a una serie de factores distintos, tanto de naturaleza histórica y biogeográfica, como a las barreras fenológicas y ecológicas al flujo génico. Las diferencias microclimáticas representan una fuente de variabilidad adicional, ya que: (1) amplifican las diferencias fenológicas de floración entre poblaciones situadas a distintas altitudes o exposiciones, y (2) provocan mosaicos espaciales en la distribución y abundancia de los polinizadores. La persistencia de estas condiciones a lo largo del tiempo (una pared rocosa orientada al norte es una nevera, mientras que una pared rocosa orientada al sur es un solarium) es muy importante, tanto en la generación de variabilidad, cómo en el mantenimiento de dicha variabilidad durante períodos largos de tiempo (Larson et al. 2000). 


\section{IMPLICACIONES PARA LA CONSERVACIÓN}

Parece claro, por tanto, que las diferencias entre escenarios ecológicos donde aparece esta planta carnívora endémica tienen importancia desde el punto de vista de la conservación de la biodiversidad, ya que el grado de diferenciación va a depender tanto de la distancia geográfica entre poblaciones, como del grado de similitud microclimática entre los diferentes escenarios ecologicos: mayores distancias, y mayores diferencias ecológicas provocarán mayores diferencias entre poblaciones, y viceversa: menores distancias, y microclimas más parecidos, más similares serán los sistemas planta-animal (Ellison et al. en prensa). Cada sector de pared, dependiendo de que esté al sol o a la sombra, tendrá a unas plantas con unos rasgos fenotípicos y fenológicos distintos (de sol, o de sombra), interaccionando con muy distintos polinizadores (de sol, o de sombra), y, en general, con muy distintos animales (presas, cleptoparásitos). Por ello, la conservación de $P$. vallisneriffolia (y por ende, de muchas otras especies de plantas rupícolas con ecología similar) pasa por mantener esta riqueza de escenarios (es decir, mantener poblaciones de sol vs de sombra), como parte de la variabilidad total a conservar. La importancia de esta aproximación, que explícitamente reconoce la heterogeneidad ambiental a distintas escalas es, a la vez, ecológica, y potencialmente evolutiva (Ellison et al. en prensa).

\section{ESTRUCTURACIÓN MICROCLIMATICA DE LAS POBLACIONES: CONSEQUENCIAS DEMOGRÁFICAS}

\section{Diferencias entre poblaciones}

Las poblaciones de $P$. vallisneriffolia muestran también diferentes abundancias y estructuras demográficas dependiendo del microhábitat donde crecen las plantas. Los muestreos de abundancia y estructura demográfica en cada población se realizaron en un sector de pared accesible, donde se mapeó de forma permanente la distribución espacial de todas las plantas, marcando individualmente a 30 plantas reproductoras. Dentro del sector de pared marcado, se contabilizaron todas las plantas establecidas, diferenciándose las siguientes fases demográficas: (1) plántula del año, (2) prereproductor (juvenil, planta que aún no ha alcanzado el umbral de tamaño por encima del cual habitualmente florecen), (3) vegetativo (planta con tamaño de reproductor potencial, pero que no ha florecido), (4) reproductor que ha producido de 13 flores, y (5) reproductor que ha desarrollado más de tres flores (ver Tabla 1). Los muestreos demográficos estuvieron muy condicionados por la difi-

TABLA 1

Estructura demográfica observada en 4 poblaciones de $P$. vallisneriifolia localizadas en el Parque Natural de Cazorla, Segura y las Villas. La humedad de la pared rocosa es del $0 \%$ para las dos poblaciones secas, y de $100 \%$ para las dos poblaciones húmedas durante todo el

período de estudio (1994-2000). Los muestreos se realizaron en Julio de 1994, y de 2000. Véase texto para los detalles metodológicos, y clasificación demográfica. Los datos corresponden a la abundancia de plantas (ind $\mathrm{m}^{-2}$ ), y al porcentaje de plantas por fase demográfica (entre paréntesis)

Demographic structure of four P. vallisneriifolia populations located in the Parque Natural de Cazorla, Segura y Las Villas. The degree of wetness of the rocky substrate is $0 \%$ in the dry populations (Poblaciones secas), and $100 \%$ in the wet populations (Poblaciones húmedas). Field work were carried out in July 1994, and 2000 (see text for methodological details and demographic classification). Figures correspond to the mean number of plants $\mathrm{m}^{-2}$, and the percentage of plants per demographic stage (in brackets)

\begin{tabular}{lllllllll}
\hline \multirow{2}{*}{$\begin{array}{l}\text { Estructura } \\
\text { demográfica }\end{array}$} & \multicolumn{3}{c}{ Poblaciones secas } & \multicolumn{3}{c}{ Poblaciones húmedas } \\
& \multicolumn{2}{c}{ Guazalmanco } & \multicolumn{2}{c}{ Agracea } & \multicolumn{2}{c}{ Escaleruela } & \multicolumn{2}{c}{ Covacho } \\
\cline { 2 - 10 } & $(1994)$ & $(2000)$ & $(1994)$ & $(2000)$ & $(1994)$ & $(2000)$ & $(1994)$ & $(2000)$ \\
\hline Plántulas & $2,25(5,3)$ & $0,5(1,5)$ & 0 & 0 & $86,9(34,1)$ & $220(58,4)$ & $67,5(28,3)$ & $381(72,3)$ \\
Pre-reproductores & $11,25(26,5)$ & $0,5(1,5)$ & $8,7(6,1)$ & $16(13,2)$ & $69,5(27,3)$ & $34,8(9,2)$ & $18,7(7,8)$ & $37,5(7,1)$ \\
Vegetativos & $15(35,3)$ & $22,5(66,2)$ & $53,3(37,3)$ & $82(67,9)$ & $18,3(7,2)$ & $17,4(4,6)$ & $15(6,3)$ & $10(1,9)$ \\
Reproductores (1-3 flores) & $14(32,9)$ & $10,5(30,8)$ & $80(56,1)$ & $22,7(18.8)$ & $69,6(27,3)$ & $86,9(23,1)$ & $112,5(47,1)$ & $55(10,4)$ \\
Reproductores (>3 flores) & 0 & 0 & $0,7(0,5)$ & 0 & $10,4(4,1)$ & $17,4(4,6)$ & $25(10,5)$ & $43,7(8,3)$ \\
Total & 42,5 & 34 & 142,7 & 120,7 & 254,7 & 376,5 & 238,7 & 527,2 \\
\hline
\end{tabular}


cultad de acceso a las paredes verticales donde crecen las plantas, y por el reducido tamaño poblacional, lo que limitó la obtención de réplicas por población. Los muestreos se realizaron en cuatro poblaciones que son representativas del rango de condiciones en las que aparece $P$. vallisneriifolia (básicamente, de humedad del substrato rocoso, y de exposición), cuando las plantas habían alcanzado el máximo crecimiento vegetativo (mayo-julio de 1994 y de 2000).

En las poblaciones que crecen en sustratos secos y soleados, el número total de plantas disminuye desde 1994 a 2000 (Poblaciones secas en Tabla 1). Apenas aparecen plántulas, ni tampoco plantas reproductoras grandes (de más de 3 flores). Además, la tendencia desde 1994 hasta el año 2000 es hacia un incremento en la proporción de plantas vegetativas, que son individuos que eran reproductores en 1994, y que han regresionado hasta esta fase por disminución de tamaño de la planta (Tabla 1). Esta tendencia, unido al casi nulo reclutamiento vía plántula, ofrece una proyección temporal de crecimiento negativo, resultando una población cada vez mas pequeña, formada predominantemente por individuos vegetativos.

Por el contrario, las poblaciones que crecen sobre roca rezumante, con suficiente luz, han aumentado incluso la densidad (sobre todo por la mayor abundancia de plántulas, ya que la primavera del 2000 fue mucho más lluviosa que la del 1994) y ofrecen una estructura demográfica opuesta a la anterior: hay gran número de plántulas, y de individuos reproductores, incluso de más de tres flores (poblaciones húmedas en Tabla 1). Por el contrario, el número de individuos en fase vegetativa es muy bajo, al contrario que en los sectores secos (Tabla 1). Por tanto, el factor grado de humedad del substrato aparece claramente como condicionante de la estructura demográfica poblacional y su variación temporal.

Diferencias entre microhábitats dentro de población

Dentro de la población Covacho del Aire, situada en el centro del área de distribución de la especie, determiné la estructura demográfica diferenciando tres tipos de microhábitats según niveles de humedad y radiación donde las plantas crecen en la pared de roca (véase Zamora 1995 y Zamora et al. 1998 para descripción de variables abióticas), y añadiendo también el suelo adyacente a la pared como un microhábitat más. Como se observa en la Tabla 2, los tres microhábitats de la pared (soleada, en sombra y con sombra fuerte), a pesar de su proximidad espacial (unos metros de distancia

TABLA 2

Estructura demográfica observada en cuatro microhábitats de la misma población (Covacho,

Sierra de Cazorla) de P. vallisneriifolia. La humedad de la pared rocosa es del $0 \%$ en el microhábitat Pared Soleada, y del $100 \%$ para los tres restantes microhábitats (Pared Sombra, Pared Sombra Fuerte y Suelo Sombra) durante todo el período de estudio (1994-2000). Los niveles de radiación total (Watios $\mathrm{m}^{-2}$ ) que llegan a cada microhábitat son: Pared Soleada:

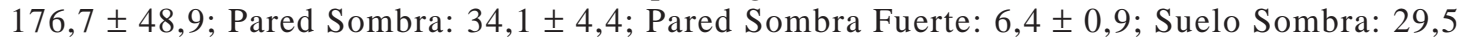
$\pm 4,5$. Los datos corresponden a valores promedio desde el amanecer hasta el atardecer, durante un día soleado (ver más información sobre las características microclimáticas en Zamora (1995) y Zamora et al. (1998). Ver texto para los detalles metodológicos, y clasificación demográfica. Los datos corresponden a la abundancia de plantas (ind $\mathrm{m}^{-2}$ ) y al porcentaje de plantas por fase demográfica (entre paréntesis)

Demographic structure of four P. vallisneriifolia subpopulations in the same location (Covacho, Sierra de Cazorla).

The degree of substrate wetness is $0 \%$ in the microhabitats "Pared soleada", and $100 \%$ in the other three, wet microhabitats (Pared sombra, Pared sombra fuerte, and Suelo sombra). Levels of total irradiance (in Watts $\mathrm{m}^{-2}$ ) were: Sunny habitat: $176.7 \pm 48.9$; Shade habitat: $34.1 \pm 4.4$; Deep shade: $6.4 \pm 0.9$; Ground shade: $29.5 \pm 4.5$ (see Table 1 for demographic classificacion, and Zamora 1995 and Zamora et al. 1998 for methodological details)

\begin{tabular}{lllllllll}
\hline Estructura demográfica & \multicolumn{2}{c}{ Pared soleada } & \multicolumn{2}{c}{ Pared Sombra } & \multicolumn{2}{c}{ Pared Sombra Fuerte } & \multicolumn{2}{c}{ Suelo Sombra } \\
& $(1994)$ & $(2000)$ & $(1994)$ & $(2000)$ & $(1994)$ & $(2000)$ & $(1994)$ & $(2000)$ \\
\hline Plántulas & 0 & $3(5,4)$ & $67,5(28,3)$ & $381(72,3)$ & $45(23,1)$ & $330(68,9)$ & $16,7(17,9)$ & $33,3(41,2)$ \\
Pre-reproductores & $15(20)$ & $8(14,5)$ & $18,7(7,8)$ & $37,5(7,1)$ & $50(25,6)$ & $52(10,8)$ & $16,7(17,9)$ & $10,8(13,3)$ \\
Vegetativos & $35(46,7)$ & $33(60)$ & $15(6,3)$ & $10(1,9)$ & $50(25,6)$ & $47(9,8)$ & $10(10,7)$ & $30(37,1)$ \\
Reproductores (1-3 flores) & $15(20)$ & $11(20)$ & $112,5(47,1)$ & $55(10,4)$ & $50(25,6)$ & $50(10,4)$ & $37,5(40,1)$ & $6,7(8,3)$ \\
Reproductores (>3 flores) & 0 & 0 & $25(10,5)$ & $43,7(8,3)$ & 0 & 0 & $12,5(13,4)$ & 0 \\
Total & 75 & 55 & 238,7 & 527,2 & 195 & 479 & 93,4 & 80,8 \\
\hline
\end{tabular}


TABLA 3

Estructura demográfica dependiendo de la fase sucesional: bajo herbáceas colonizadoras (Bajo Plantas) y en los espacios abiertos entre plantas colonizadoras (Entre Plantas). Ver texto para

los detalles metodológicos, y clasificación demográfica. Los datos corresponden a la abundancia (ind $\mathrm{m}^{-2}$ ) y al porcentaje de plantas por fase demográfica (entre paréntesis) de las poblaciones Río Madera (Población 1), y Barranco Iglesias (Población 2) del Parque Natural de Cazorla, Segura y Las Villas

\begin{tabular}{|c|c|c|c|c|}
\hline \multirow{2}{*}{ Estructura demográfica } & \multicolumn{2}{|c|}{ Población 1} & \multicolumn{2}{|c|}{ Población 2} \\
\hline & Entre Plantas & Bajo Plantas & Entre Plantas & Bajo Plantas \\
\hline Plántulas & $30(78,7)$ & $7(97,2)$ & $80(66,9)$ & $2(45,4)$ \\
\hline Pre-reproductores & $5(13,1)$ & $0,2(2,8)$ & $25(20,9)$ & $0,4(9,1)$ \\
\hline Vegetativos & $1(2,6)$ & 0 & $9(7,6)$ & $2(45,4)$ \\
\hline Reproductores (1-3 flores) & $2(5,2)$ & 0 & $4,2(3,5)$ & 0 \\
\hline Reproductores ( $>3$ flores) & $0,1(0,2)$ & 0 & $1,3(1,1)$ & 0 \\
\hline Total & 38,1 & 7,2 & 109,5 & 4,4 \\
\hline
\end{tabular}

entre sí), muestran estructuras demográficas muy distintas. En el microhábitat Pared Soleada, el número de plantas disminuye en el periodo 19942000. La sequedad del substrato rocoso limita considerablemente el establecimiento de plántulas, y el desarrollo de individuos reproductores de gran tamaño, dominando los individuos vegetativos, que representan más de la mitad de la población.

Los individuos reproductores también representan una pequeña fracción (10-25\%) del total de plantas en el microhábitat Pared sombra fuerte (Tabla 2). Por el contrario, este hábitat, húmedo y muy sombreado, favorece el establecimiento de las plántulas. Sin embargo, aparece una limitación en el crecimiento vegetativo debido a la escasez de luz, y por tanto, no aparecen plantas reproductoras de gran tamaño (Zamora et al. 1998). Sólo aparecen plantas grandes, acompañadas de gran número de plántulas, y muy pocas plantas en fase vegetativa, cuando, a una elevada humedad del substrato rocoso, se une una cantidad de luz suficiente, como ocurre en el microhábitat Pared Sombra. Este patrón demográfico entre microhábitats secos y húmedos dentro de población (Tabla 2), reproduce fielmente el observado entre poblaciones secas y húmedas (Tabla1).

\section{Diferencias dependiendo de la sucesión vegetal en taludes}

Por el contrario, en la poblaciones situadas en el microhábitat suelo al pié de pared (Suelo som- bra), la densidad de plantas reproductoras disminuye desde 1994 hasta el 2000, desapareciendo todas las plantas reproductoras grandes, e incrementando la proporción de individuos vegetativos (Tabla 2). Las condiciones abióticas (radiación, humedad del substrato) no cambiaron en el tiempo, el único cambio apreciable fue la colonización por herbáceas del suelo rocoso donde crecían las carnívoras, de manera que en 1994, la cobertura de herbáceas en la parcela marcada era del $0 \%$, mientras que en el 2000 , la cobertura era del $35 \%$.

Para comprobar si el efecto de la invasión de herbáceas afectaba de forma diferencial a las distintas fases demográficas de la planta, realicé en junio del 2000 muestreos de plantas en los taludes al pié de las paredes donde crecen las plantas carnívoras, diferenciando tres tipos de microhábitats: (1) microhábitat herbazal recién establecido (Bajo Plantas en Tabla 3), y (2) microhábitat espacios entre macollas de plantas (Entre Plantas en Tabla 3), todavía no colonizados, y (3) microhábitat herbazal antiguo y denso, establecido hace años.

En los espacios abiertos entre las herbáceas colonizadoras aparecen plántulas, acompañadas de un número muy reducido de plantas pertenecientes a todas las fases demográficas (microhábitat Entre Plantas, Tabla 3). Sin embargo, bajo las hierbas competidoras recién establecidas, prácticamente desaparecen las formas adultas, y apenas quedan plántulas (microhábitat Bajo Plantas, Tabla 3). Cuando el herbazal lleva ya más de un año establecido, no quedan bancos de plántulas 
bajo las hierbas competidoras, ni formas de resistencia vegetativa. La conclusión es simple: el herbazal excluye completamente a la población de $P$. vallisneriffolia del microhábitat que podría ser óptimo para esta carnívora, los taludes rocosos al pié de las paredes rezumantes.

\section{VIABILIDAD DE LAS POBLACIONES A LARGO PLAZO}

El habitat óptimo para $P$. vallisneriffolia, por tanto, es aquel que tiene agua "ad libitum", que recibe una cantidad suficiente de radiación, y que está libre de competencia con otras plantas herbáceas (Zamora et al. 1996, Zamora et al. 1998). Sin embargo, esta combinación ideal de factores no es común en el ambiente mediterráneo, más aún cuando el incremento de aridez está provocando una disminución del tamaño de las "islas húmedas" aprovechables por P. vallisneriffolia, cada vez más rodeadas por un mar de sequía estival. Este problema es especialmente relevante en el contexto de la sucesión ecológica, ya que, bajo las condiciones climáticas actuales, los hábitats húmedos que presentan un mínimo de suelo son rápidamente colonizados por otras plantas ávidas de agua (el principal factor limitante en el área mediterránea), que desplazan competitivamente a $P$. vallisneriifolia por sobrecrecimiento. El único hábitat que les queda libre de la competencia con otras plantas son las paredes verticales húmedas, carentes de suelo, donde otras plantas con sistemas radiculares más desarrollados no pueden medrar. El hábito carnívoro de $P$. vallisneriffolia puede considerarse como una preadaptación provechosa al permitir la colonización y mantenimiento de poblaciones viables a lo largo del tiempo en las substratos rocosos, donde la captación de nutrientes via raices está muy limitada. Como resultado de vivir en estas condiciones extremas, $P$. vallisneriifolia parece haber desarrollado una mayor dependencia de las presas animales como fuente de nutrientes para su crecimiento y reproducción que otras especies del género Pinguicula que crecen en suelo (Zamora et al. 1997).

El carácter perenne de esta planta le permite ralentizar la extinción de las poblaciones, incluso en ausencia de reclutamiento vía plántula (e.g., lugares secos y soleados), o incluso cuando su crecimiento vegetativo y desarrollo reproductor está muy limitado (e.g., lugares muy sombreados). En este contexto, la capacidad de la planta para realizar progresiones demográficas regresivas (de reproductor a vegetativo), unido a las vías vegetativas de propagación (estolones y yemas axilares) permiten la persistencia vegetativa de los individuos hasta que la fuente de agua se agote, lo que puede suponer un proceso de declive y extinción de la población muy lentos (poblaciones remanentes sensu Eriksson 1996, García et al. 2000).

Por el contrario, la reproducción sexual vía semilla representa la lotería de búsqueda de nuevos espacios abiertos húmedos. Sin embargo, con la tendencia hacia una mayor aridez actual, los escasos micrositios adecuados para la germinación y establecimiento de plántulas se desplazan hacia los sectores de las paredes más umbríos y húmedos, pero donde el crecimiento y desarrollo reproductivo está muy limitado por la escasez de luz, por lo que sólo una pequeña fracción de las plántulas que se establecen llegan a reproductores. El resultado es que estos microhabitats húmedos, pero muy umbríos, se convierten en "sumideros poblacionales atractivos", donde pueden entrar y establecerse con éxito muchos más propágulos de los que potencialmente se pueden generar vía reproducción dentro del sumidero.

\section{CONSECUENCIAS PARA LA CONSERVACIÓN DE LAS PLANTAS CARNÍVORAS}

Si se mantiene la actual tendencia hacia una progresiva aridez climática, agravada por el aumento en la irregularidad de las precipitaciones, y mayor frecuencia de los episodios de sequía en la cuenca mediterránea (Rodó \& Comín 2001, Peñuelas 2001), es muy previsible también que: (1) se acelere la reducción en la extensión de las zonas húmedas permanentes de montaña existentes en la actualidad, y (2) se limite considerablemente la aparición de nuevas zonas húmedas. Como consecuencia, un pronóstico verosimil sobre el futuro de las poblaciones de $P$. vallisneriifolia a medio y largo plazo, que se apoya en la tendencia observada en el período 1994-2000, es que: (1) las poblaciones que están en paredes secas y soleadas reducirán aún más sus efectivos, o incluso desaparecerán, (2) El reclutamiento poblacional sólo será posible, como ocurre en la actualidad, en las pocas paredes rezumantes que todavía quedan, cuyos sectores más umbríos pueden convertirse en una trampa para la viabilidad de las poblaciones a largo plazo por la limitación de luz, ("sumideros poblacionales atractivos"), y (3) las bases de las paredes y los taludes de travertino, muy húmedos y con suficiente luz, se están convirtiendo en herbazales muy densos, que excluyen competitivamente a las carnívoras, como se ha comprobado en la mayoría de las poblaciones en tan solo 7 años (19942000). Este problema ocurre no solo con $P$. vallisneriifolia, sino también con las otras dos 
especies carnívoras que aparecen en el mismo tipo de hábitats rocosos mediterráneos en la $\mathrm{Pe}$ nínsula Ibérica: $P$. mundii, y $P$. submediterránea (Zamora et al. 1996).

La invasión de hierbas se ha visto favorecida por la construcción de cercados cinegéticos al pié de las paredes para excluir a los grandes herbívoros, con la supuesta finalidad de proteger, además de a $P$. vallisneriffolia, a otras especies endémicas que suelen crecer en la base de los taludes rocosos (e.g., Viola cazorlensis, Aquilegia cazorlensis). En realidad, lo que favorecen estas exclusiones de herbívoros es el desarrollo de herbazales muy densos, que excluyen a las carnívoras (que, por cierto, apenas son comidas por los herbívoros) y a las otras especies endémicas.

Para la conservacion de estas especies de carnívoras, hacen falta llevar a cabo las siguientes medidas de manejo:

(1) Crear artificialmente espacios libres de plantas competidoras arrancando periódicamente las herbáceas que crecen en los taludes, y al pié de las paredes rezumantes, donde $P$. vallisneriffolia puede establecer poblaciones viables. Lo ideal sería generar un sistema de parches libres de vegetación, simulando las perturbaciones naturales (desprendimientos de rocas, avenidas de agua, pisoteo de herbívoros ungulados, etc.), donde las carnívoras se establecen con rapidez. Estos manejos requieren un seguimiento periódico, con el fin de mantener todos los años un número mínimo de nichos de regeneración efectivos para las poblaciónes de Pinguicula.

(2) Dichas medidas de manejo deberían complementarse con reintroducciones de la especie, a partir de semillas y yemas invernales de resistencia, en aquellas paredes que permanecen húmedas durante todo el verano, y a las que no ha llegado la colonización natural vía semilla, por la fragmentación y aislamiento en el que se encuentran las poblaciones. Se deberían utilizar siempre en estas reintroducciones propágulos provenientes de poblaciones geográficamente próximas (la más cercana), y ecológicamente más similar (misma exposición) a la pared de roca donde se pretende realizar la reintroducción.

\section{AGRADECIMIENTOS}

Agradezco a Anna Traveset y a Mary Kalin Arroyo la invitación a participar en el taller "Aspectos teóricos de la reproducción en plantas, consequencias para la conservación”, celebrado en Palma de Mallorca, y financiado por la CYTED, a través de la Red de Ecosistemas Mediterráneos, Subprograma de Biodiversidad. Jose Ramón Obe- so, Jose Máría Gómez y un revisor anónimo aportaron provechosas sugerencias a una versión preliminar del manuscrito. A Rodrigo Medel, por su trabajo editorial. La Dirección del Parque Natural de Cazorla, Segura y las Villas autorizó y facilitó el trabajo de campo.

\section{LITERATURA CITADA}

BLANCA G, M CUETO, MJ MARTÍNEZ-LIROLA \& J MOLERO-MESA (1998) Threatened vascular flora of Sierra Nevada (southern Spain). Biological Conservation 85: 269-285.

BLANCA G, B CABEZUDO, JE HERNÁNDEZ-BERMEJO, CM HERRERA, J MUÑOZ \& B VALDES (2000) Libro rojo de la flora silveste amenazada de Andalucía. Tomo II: especies vulnerables. Editorial Junta de Andalucía, Sevilla, España. 375 pp.

BLONDEL J \& J ARONSON (1999) Biology and wildlife of the mediterranean region. Oxford University Press, Oxford, United Kingdom. 328 pp.

BRONSTEIN JL (1994) Conditional outcomes in mutualistic interactions. Trends in Ecology and Evolution 9: 214-217.

CASPER SJ (1972) Pinguicula L. En: Tutin TG, VH Heywood, NA Burges, DM Moore, DH Valentine, SM Walters \& DA Webb (eds) Flora europaea 3: 294296. Cambridge University press, Cambridge, United Kingdom.

CASTROVIEJO S (1997) The flora Iberica project: results and problems. Lagascalia 19: 371-380.

CORBET SA (1990) Pollination and the weather. Israel Journal of Botany 39: 13-30.

ELLISON A, MNJ GOTELLI, JS BREWER, L COCHRANSTAFIRA, J KNEITEL, TE MILLER, AS WORLEY \& R ZAMORA (en prensa) Carnivorous plants as model systems for ecological research. Advances in Ecological Research.

ERIKSSON O (1996) Regional dynamics of plants: a review of evidence for remnant, source-sink and metapopulations. Oikos 77: 248-258.

FALK DA \& KE HOLSINGER (1991) Genetics and conservation of rare plants. Oxford University Press, New York, New York. 283 pp.

FERNÁNDEZ-CASAS J \& A CEVALLOS-JIMÉNEZ (1982) Plantas silvestres de la Península Ibérica rupicolas. Editorial Blume, Barcelona, España. 432 $\mathrm{pp}$.

GARCÍA D, R ZAMORA, JA HÓDAR \& JM GÓMEZ (1999) Age structure of Juniperus communis L. in the Iberian peninsula: conservation of remnant populations in Mediterranean mountains. Biological Conservation 87: 215-220.

GIVNISH TJ (1989) Ecology and evolution of carnivorous plants. En: Abrahamson WG (ed) Plant-animal interactions: 242-290. McGraw-Hill Book Company, New York, New York.

GÓMEZ-CAMPO C \& JM HERRANZ-SANZ (1993) Conservation of Iberian endemic plants: the botanical reserve of La Encantada (Villarrobledo, Albacete, Spain). Biological Conservation 64: 155-160. 
HERRERA CM (1990) Daily patterns of pollinator activity, differential pollinating effectiveness, and floral resource availability in a summer flowering Mediterranean shrub. Oikos 58: 277-288.

HERRERA CM (1995a) Floral biology, microclimate, and pollination by ectothermic bees in an early-blooming herb. Ecology 76: 218-228.

HERRERA CM (1995b) Microclimate and individual variation in pollinators: flowering plants are more than their flowers. Ecology 76: 1516-1524.

HERRERA CM (1997) Thermal biology and foraging responses of insect pollinators to the forest floor irradiance mosaic. Oikos 78: 601-611.

JOHANNESSON K (2001) Parallel speciation: a key to sympatric divergence. Trends in Ecology and Evolution 16: 148-153.

JUNIPER BE, RB ROBINS \& DM JOEL (1989) The carnivorous plants. Academic Press, London, United Kingdom. 353 pp.

LARSON DW, U MATTHES \& PE KELLY (2000) Cliff ecology. Cambridge University Press, Cambridge, United Kingdom. 233 pp.

LINHART YB \& MC GRANT (1996) Evolutionary significance of local genetic differentiation in plants. Annual Review of Ecology and Systematics 27: 237277.

PEÑUELAS J (2001) Cambios atmosféricos y climáticos y sus consecuencias sobre el funcionamiento y la estructura de los ecosistemas terrestres mediterráneos. En: Zamora R \& FI Pugnaire (eds) Ecosistemas mediterráneos: análisis funcional: 423-463. Serie de Textos Universitarios No. 32, Servicio de Publicaciones del CSIC, Granada, España.

RODÓ X \& F COMÍN (2001) Fluctuaciones del clima mediterráneo: conexiones globales y consecuencias regionales. En: Zamora R \& FI Pugnaire (eds) Ecosistemas mediterráneos: análisis funcional: 1-35. Serie de Textos Universitarios No. 32, Servicio de Publicaciones del CSIC, Granada, España.
SCHEMSKE DW, BC HUSBAN, MH RUCKELSHAUS, C GOODWILLIE, IM PARKER \& J BISHOP (1994) Evaluating approaches to the conservation of rare and endangered plants. Ecology 75: 584-606.

SCHLUTER D (1998) Ecological causes of speciation. En: Howard DJ \& SH Berlorcher (eds) Endless formsspecies and speciation: 114-129. Oxford University Press, Oxdford, United Kingdom.

STUDNICKA M (1991) Interesting succulent features in the Pinguicula species from the Mexican evolutionary centre. Folia Geobotanica et Phytotaxonomica 26: 459-462.

ZAMORA R (1995) The trapping success of a carnivorous plant (Pinguicula vallisnerifolia): the cumulative effects of availability, attraction, retention, and robbery of prey. Oikos 73: 309-322.

ZAMORA R (1999) Conditional outcomes of interactions: the pollinator-prey conflict of an insectivorous plant. Ecology 80: 786-795.

ZAMORA R \& JM GÓMEZ (1996) Carnivorous plantslug interaction: a trip from herbivory to kleptoparasitism. Journal of Animal Ecology 65:154160.

ZAMORA R, M JAMILENA, MR REJÓN \& G BLANCA (1996) Two new species of the carnivorous genus Pinguicula (Lentibulariaceae) from Mediterranean habitats. Plant Systematics and Evolution 200: 41-60.

ZAMORA R, JM GÓMEZ \& J HÓDAR (1997) Responses of a carnivorous plant to prey and inorganic nutrients in a Mediterranean environment. Oecologia 111: 443451.

ZAMORA R, JM GÓMEZ \& J HÓDAR (1998) Fitness responses of a carnivorous plant in contrasting ecological scenarios. Ecology 79: 1630-1644. 